\title{
ANÁLISE ANTIGÊNICA E MOLECULAR DE AMOSTRAS CITOPÁTICAS DO VÍRUS DA DIARRÉIA VIRAL BOVINA ${ }^{1}$
}

\author{
ANTIGENIC AND MOLECULAR ANALYSIS OF CYTOPATHIC \\ ISOLATES OF BOVINE VIRAL DIARRHEA VIRUS
}

\author{
Fernando Luiz Tobias ${ }^{2}$ Anselmo Odeon ${ }^{3}$ Edwiges Maristela Pituco ${ }^{4}$ \\ Rudi Weiblen ${ }^{5}$ Dino César Garcez ${ }^{6}$ Eduardo Furtado Flores ${ }^{7}$
}

RESUMO

Sete amostras citopáticas do vírus da Diarréia Viral Bovina (BVDV) isoladas de casos clínicos e do sangue de bezerros de rebanhos com problemas reprodutivos foram analisadas. Todas as amostras caracterizadas possuíam uma mistura de vírus citopáticos ( $\mathrm{cp}$ ) e não-citopáticos (ncp), que foram clonados biologicamente, originando populações puras de vírus de cada biotipo. Os clones cp e ncp obtidos foram caracterizados antigenicamente com um painel de anticorpos monoclonais (MAbs) $e$ quanto à expressão da proteína não-estrutural NS3. A análise de reconhecimento pelos MAbs revelou dois padrões de reatividade: 1. Em cinco casos, os vírus cp e ncp de uma mesma amostra mostraram-se antigenicamente muito semelhantes entre si, indicando tratar-se de verdadeiros "pares" de vírus, nos quais o vírus cp origina-se do ncp através de mutações ou recombinações; 2. Duas amostras, no entanto, continham vírus cp e ncp com diferenças antigênicas consideráveis entre si. A análise de polipeptídeos não-estruturais das amostras ncp através de Western immunoblot revelou uma única banda de reatividade, de massa aproximada de $125 \mathrm{kDa}$, correspondente à proteína nãoestrutural NS23. As amostras cp expressaram, além da NS23, um polipeptídeo de massa aproximada de $80 \mathrm{kDa}$, correspondente à NS3. Duas amostras cp apresentaram diferenças na migração da NS23. Uma amostra apresentou a NS23 com massa menor do que $125 \mathrm{kDa}$, enquanto outra amostra apresentou duas bandas de reatividade, com massas menor e maior que a NS23 dos demais vírus, respectivamente. Esses resultados confirmam achados anteriores de que amostras de campo citopáticas do BVDV geralmente possuem vírus dos dois biotipos e que o fenótipo citopático está associado à expressão da proteína NS3. O isolamento de amostras citopáticas do sangue de animais clinicamente normais e de um feto, no entanto, demonstra que a ocorrência de vírus cp não se restringe à casos da Doença das Mucosas.

Palavras-chave: vírus da diarréia viral bovina, $B V D V$, análise antigênica, citopatologia.

\section{SUMMARY}

Seven cytopathic isolates of bovine viral diarrhea virus (BVDV) isolated from clinical cases and blood of calves from herds with reproductive problems were analysed. All isolates contained a mixture of cytopathic ( $\mathrm{cp}$ ) and noncytopathic (ncp) viruses which were biologically cloned yielding pure populations of viruses of either biotype. The cp and ncp viruses obtained by cloning were characterized antigenically with a panel of monoclonal antibodies (MAbs) and regarding to the expression of the non-structural polypeptide NS3. The analysis of $M A b$ binding revealed two patterns of reactivity: 1 . In five isolates, the $\mathrm{cp}$ and ncp viruses from the same isolate were antigenically very similar to each other, suggesting they represent true "pairs"of viruses. 2. Two isolates, however, yielded $\mathrm{cp}$ and ncp viruses antigenically different from each other. Western immunoblot analysis of non-structural polypeptides of ncp viruses revealed a unique band of reactivity, with a mass of approximately $125 \mathrm{kDa}$, corresponding to the NS23 of the standard BVDV Singer strain. In addition to NS23, the cp viruses expressed a polypeptide of approximatelly $80 \mathrm{kDa}$, corresponding to the NS3. The NS23 of two cp viruses displayed an altered migration in SDS-PAGE compared to the other viruses. In one virus, the NS23 had a molecular mass lower than expected whereas in other virus, two bands of reactivity were observed:

\footnotetext{
${ }^{1}$ Trabalho realizado com auxílio financeiro do CNPq/FINEP/MCT (PRONEX em Virologia Veterinária, 215/96) e parte da Dissertação de Mestrado do primeiro autor. Programa de Pós-graduação em Medicina Veterinária. Universidade Federal de Santa Maria (UFSM). ${ }^{2}$ Médico Veterinário, Mestrando em Medicina Veterinária. UFSM. Bolsista da CAPES.

${ }^{3}$ Médico Veterinário, PhD, Instituto Nacional de Tecnologia Agropecuária - INTA. Balcarce, Província de Buenos Aires, Argentina. ${ }^{4}$ Médico Veterinário, Doutor, Instituto Biológico de São Paulo, São Paulo.

${ }^{5}$ Professor Titular, PhD, Departamento de Medicina Veterinária Preventiva (DMVP), eDepartamento deMicrobiologia e Parasitologia(DMP). UFSM. Bolsista doCNPq

${ }^{6}$ Acadêmico do Curso de Medicina Veterinária, UFSM. Bolsista de Iniciação Científica, CNPq.

${ }^{7}$ Professor Adjunto, PhD, DMVPe DMP, UFSM, 97105-900, Santa Maria, RS. Bolsista do CNPq. E-mail: Flores@ccr.ufsm.br. Autor para correspondência. 
one smaller and another bigger than the standard NS23, respectively. Our findings confirm previous results that cytopathic $B V D V$ field isolates usually contain a mixture of viruses of both biotypes and that cytopathogenicity correlates with the expression of NS3. The isolation of $c p$ viruses from the blood of clinically normal cattle, however, demonstrates that their occurrence is not restricted to cases of mucosal disease.

Key words: bovine viral diarrhea virus, $B V D V$, antigenic diversity, cytopathology.

\section{INTRODUÇÃO}

O vírus da Diarréia Viral Bovina (bovine viral diarrhea virus, BVDV) é considerado um dos patógenos mais importantes de bovinos e está distribuído mundialmente (BOLIN, 1995). O BVDV é um vírus pequeno (40-60nm de diâmetro), genoma RNA de polaridade positiva, de aproximadamente 12.5 kilobases (HORZINEK, 1981; COLLETT et al., 1989), classificado na família Flaviviridae, gênero Pestivírus (FRANCKI et al., 1991). A infecção pelo BVDV pode estar associada a várias manifestações clínicas que variam desde infecções subclínicas até formas clínicas fatais (BROWNLIE, 1990; BAKER, 1995; BOLIN, 1995).

Amostras de campo de BVDV podem ser classificadas em biotipos citopático (cp) e nãocitopático (ncp) de acordo com o efeito de sua replicação em cultivo celular (GILLESPIE, 1962; McCLURKIN et al., 1985). As amostras ncp representam a grande maioria da amostras de campo e estão associadas às diversas manifestações clínicas da infecção (BOLIN, 1995; DUBOVI, 1998). Amostras cp constituem uma minoria e são isoladas quase que exclusivamente de animais afetados pela Doença das Mucosas (DM) (BROWNLIE, 1990; BOLIN, 1995). A infecção de fêmeas gestantes entre os dias 40 e 120 de gestação com amostras ncp é freqüentemente seguida de infecção fetal com indução de imunotolerância (BROWNLIE, 1990). Fetos infectados nesse período nascem persistentemente infectados (PI). Em animais PI, é comum o desenvolvimento de uma enfermidade aguda e fatal chamada de Doença das Mucosas (DM) (BROWNLIE, 1990; BOLIN, 1995). A etiopatogenia da DM é atribuída a uma superinfecção do animal PI com um vírus $\mathrm{cp}$, geralmente originado a partir do vírus ncp original através de mutações ou recombinações no genoma (McCLURKIN et al., 1985; MEYERS et al., 1992; DONIS, 1995). Por isso, em animais que desenvolvem essa forma clínica, é comum o isolamento de vírus dos dois biotipos (McCLURKIN et al., 1985; BROWNLIE, 1990; BOLIN, 1995). Geralmente, devido à sua origem comum, os vírus ncp e cp isolados de casos de DM são antigenicamente muito semelhantes entre si (HOWARD et al., 1987; CORAPI et al., 1988).

Os biotipos ncp e cp podem ser diferenciados em nível molecular pela expressão e processamento distintos de dois polipeptídeos virais nãoestruturais (DONIS, 1995). Amostras ncp expressam apenas um polipeptídeo de massa aproximada de $125 \mathrm{kDa}$ (NS23). Em contraste, amostras cp expressam a NS23 e também um polipeptídeo de aproximadamente $80 \mathrm{kDa}$ (NS3), correspondente à porção 2/3 carboxi-terminal da NS23 (DONIS, 1995). A geração da NS3/p80 nas amostras cp pode ocorrer pela clivagem da NS23 ou por duplicação do gene da NS3 (MEYERS et al., 1992; DONIS, 1995).

O presente estudo teve como objetivos investigar a presença de vírus dos dois biotipos em amostras de campo de BVDV que produziram citopatologia em cultivo celular e caracterizar esses vírus antigenicamente e quanto à expressão dos polipeptídeos não-estruturais NS3 e NS23.

\section{MATERIAL E MÉTODOS}

Sete amostras de BVDV que produziram efeito citopático (ECP) em cultivo celular foram analisadas. A origem e informações adicionais referentes a essas amostras estão apresentadas na tabela 1. A amostra padrão Singer foi utilizada como controle nos testes de reatividade com anticorpos monoclonais e na análise de proteínas através de Western immunoblot. As amostras foram propagadas em células de linhagem de rim bovino (Madin Darby bovine kidney, MDBK, ATCC-CCL22), cultivadas em meio essencial mínimo (MEM) com antibióticos (penicilina $16 \mathrm{mg} / \mathrm{l}$ e estreptomicina, $0,4 \mathrm{mg} / \mathrm{kg}$ ) suplementado com $5 \%$ de soro eqüino.

A presença de vírus do biotipo cp foi evidenciada por ocasião do isolamento do vírus, pela produção de ECP característico. A presença de vírus do biotipo ncp foi demonstrada através de ensaios de placa seguidos de coloração dos tapetes celulares por imunocitoquímica (ICQ), de acordo com técnica descrita por BOTTON et al. (1998).

As amostras foram inicialmente propagadas em cultivo celular e, após, clonadas biologicamente. A clonagem dos vírus $\mathrm{cp}$ foi realizada através de ensaios de placa e, a dos vírus ncp, através do método de diluição limitante (BOTTON et al., 1998). Os clones obtidos foram propagados em cultivo celular para posterior caracterização. As placas produzidas pelos vírus cp foram visualizadas diretamente nos tapetes celulares ou após coloração com cristal violeta. Os focos infecciosos dos vírus ncp foram evidenciados através de ICQ. 
Tabela 1 - Origem e informações adicionais das amostras citopáticas do vírus da Diarréia Viral Bovina (BVDV) analisadas.

\begin{tabular}{|c|c|c|c|c|}
\hline Identificação & Espécie/Curso clínico. & Biotipos & Local/Ano & Clones obtidos \\
\hline EMP 2 & $\begin{array}{l}\text { Soro. Bezerro oriundo de } \\
\text { rebanho com problemas } \\
\text { reprodutivos. }\end{array}$ & $\mathrm{cp} / \mathrm{ncp}$ & SP-Brasil, 1995 & $\begin{array}{l}\text { EMP } 2 \text { nсp } 1,2 \text { e } 3 \\
\text { EMP } 2 \text { cp } 1,2 \text { e } 3\end{array}$ \\
\hline EMP 4 & $\begin{array}{l}\text { Soro. Bezerro oriundo } \\
\text { de rebanho com proble- } \\
\text { mas reprodutivos. }\end{array}$ & $\mathrm{cp} / \mathrm{ncp}$ & $\begin{array}{l}\text { SP-Brasil, } \\
1995\end{array}$ & $\begin{array}{l}\text { EMP } 4 \text { ncp } 1,2 \text { e } 3 \\
\text { EMP } 4 \text { cp } 1,2 \text { e } 3\end{array}$ \\
\hline EMP 5 & $\begin{array}{l}\text { Soro. Bezerro oriundo de } \\
\text { rebanho com problemas } \\
\text { reprodutivos. }\end{array}$ & $\mathrm{cp} / \mathrm{ncp}$ & SP-Brasil,1995 & $\begin{array}{l}\text { EMP } 5 \text { nсp } 1,2 \text { e } 3 \\
\text { EMP } 5 \text { cp } 1,2 \text { e } 3\end{array}$ \\
\hline $34 \mathrm{P}$ & $\begin{array}{l}\text { Rim. Feto bovino } \\
\text { coletado em matadouro. }\end{array}$ & $\mathrm{cp} / \mathrm{ncp}$ & Argentina, 1997 & $\begin{array}{l}34 \text { P ncp } 1,2 \text { e } 3 \\
34 \text { P cp } 1,2 \text { e } 3\end{array}$ \\
\hline INTA 1 & $\begin{array}{l}\text { Linfonodo. Doenças das } \\
\text { Mucosas. }\end{array}$ & $\mathrm{cp} / \mathrm{ncp}$ & Argentina, 1995 & $\begin{array}{l}\text { INTA } 1 \text { ncp } 1,2 \text { e } 3 \\
\text { INTA } 1 \text { cp } 1,2 \text { e } 3\end{array}$ \\
\hline INTA 3 & $\begin{array}{l}\text { Linfonodo. Doença das } \\
\text { Mucosas. }\end{array}$ & $\mathrm{cp} / \mathrm{ncp}$ & Argentina, 1995 & $\begin{array}{l}\text { INTA } 3 \text { nсp } 1,2 \text { e } 3 \\
\text { INTA } 3 \text { cp } 1,2 \text { e } 3\end{array}$ \\
\hline INTA 4 & $\begin{array}{l}\text { Leucócitos. Novilha } \\
\text { oriunda de rebanho com } \\
\text { problemas reprodutivos. }\end{array}$ & $\mathrm{cp} / \mathrm{ncp}$ & Argentina, 1995 & $\begin{array}{l}\text { INTA } 4 \text { ncp } 1,2 \text { e } 3 \\
\text { INTA } 4 \text { cp } 1,2 \text { e } 3\end{array}$ \\
\hline
\end{tabular}

mações referentes a essas amostras estão apresentados na tabela 1 . Todas as amostras analisadas continham uma mistura de vírus dos dois biotipos (cp e ncp). A presença de vírus do biotipo cp foi evidenciada já nas primeiras passagens realizadas durante o isolamento do vírus do material clínico. A demonstração dos vírus ncp exigiu a realização da técnica de ICQ. A execução dessa técnica em tapetes celulares infectados com as amostras de campo permitiu a visualização de dois tipos de focos infecciosos, com e sem destruição celular. A clonagem biológica das amostras, através de diluição limitante ou ensaios de placa, permitiu a obtenção de pelo menos três clones de cada biotipo por amostra (tabela 1).

Amostras citopáticas representam uma minoria dos isolados de campo do BVDV e têm sido isoladas quase que exclusivamente de casos de Doença das Mucosas (BROWNLIE, 1990; RIDPATH et al., 1991; BOLIN, 1995). Várias evidências indicam que os vírus cp se originam do vírus ncp original, que infecta os

A reatividade de um painel de MAbs com os clones de vírus obtidos foi analisada através da técnica de imunofluorescência indireta (IFI), executada de acordo com protocolos-padrão (BOTTON $\boldsymbol{e t}$ al., 1998). Para isso, utilizou-se um painel de 18 MAbs específicos para as glicoproteínas E0 e E2, e para a proteína não-estrutural NS23 do BVDV cepa padrão Singer (CORAPI et al., 1990).

As proteínas virais produzidas em células infectadas foram analisadas pela técnica de Western immunoblot, utilizando o MAb 20.10.6 que reage com a porção carboxi-terminal da proteína NS23, correspondente ao polipeptídeo NS3 (CORAPI $\boldsymbol{e t}$ al., 1990). Células MDBK infectadas com cada um dos vírus foram lisadas conforme metodologia descrita por LAEMNLI (1970). A técnica de Western immunoblot para análise de polipeptídeos virais em células infectadas com o BVDV também foi descrita anteriormente (BOTTON et al., 1998).

\section{RESULTADOS E DISCUSSÃO}

Sete amostras de campo citopáticas do BVDV foram analisadas. A origem e demais infor- animais PI, através de mutações ou recombinações no genoma (HOWARD $\boldsymbol{e t}$ al., 1987; CORAPI $\boldsymbol{e t}$ al., 1988; BOLIN, 1995; DONIS, 1995). Por isso, a ocorrência do vírus cp está quase sempre associada ao vírus ncp que lhe deu origem. A transformação de vírus ncp em cp também tem sido reproduzida experimentalmente através de manipulações genéticas no RNA viral (MENDEZ et al., 1998). Amostras cp também têm sido esporadicamente isoladas de soro fetal e de animais PI imunizados com vacinas com vírus vivo formuladas com cepas cp (RIDPATH $\boldsymbol{e} t$ al., 1991; BOLIN, 1995; BOLIN \& RIDPATH, 1998). O isolamento ocasional de amostras puras de BVDV cp de soro fetal bovino também tem sido relatado, embora com uma freqüência ainda menor. Algumas dessas amostras têm sido identificadas como vírus vacinal, enquanto outras têm origem desconhecida (BOLIN \& RIDPATH, 1998). No presente estudo, duas amostras analisadas (INTA $1 \mathrm{e}$ 3) foram isoladas de casos diagnosticados clinicamente como DM. Nesses casos, o vírus cp geralmente origina-se do par ncp, embora diferenças antigênicas significativas tenham sido observadas 
entre os clones cp e ncp da amostra INTA 3. A vacinação de animais PI com vírus vivo modificado também tem sido implicada no desenvolvimento de DM. Em alguns desses casos, diferenças antigênicas consideráveis entre os vírus ncp e cp têm sido detectadas (RIDPATH et al., 1991; BOLIN, 1995).

$\mathrm{O}$ isolamento de amostras cp do sangue de animais saudáveis, oriundos de rebanhos com problemas reprodutivos (EMP-2, 4 e 5, INTA 4) e de um feto coletado em matadouro (34P) representa exceção à ocorrência de amostras cp do BVDV. Os clones cp e ncp obtidos de cada amostra foram então caracterizados com um painel de MAbs, com o objetivo de determinar as relações antigênicas e demonstrar similaridades antigênicas que pudessem explicar a provável origem dos vírus $\mathrm{cp}$ dessas amostras. Os resultados dessa análise estão apresentados na tabela 2 .

Considerando-se os três clones de ambos os biotipos purificados de cada amostra, quatro desses isolados (EMP-2, EMP-5, 34P e INTA 4), além da amostra INTA 1 , continham pelo menos um par de vírus cp e ncp antigenicamente idênticos ou muito semelhantes entre si. Os prováveis "pares" de cada amostra estão assinalados com asteriscos (tabela 2). Esse alto nível de similaridade, ou mesmo de identidade em alguns casos, contrasta com as diferenças antigênicas marcantes geralmente observadas quando diferentes isolados de campo do BVDV são comparados (CORAPI et al., 1988; 1990; BOTTON et al., 1998). Semelhanças dessa magnitude são comumente observadas quando vírus ncp e cp isolados de um mesmo animal acometido de DM são analisados antigenicamente (HOWARD et al., 1987; CORAPI et al., 1988; RIDPATH et al., 1991). Por isso, a grande similaridade antigênica observada entre alguns componentes ncp e cp das amostras mencionadas sugere que esses vírus tenham uma origem comum. A comprovação dessa hipótese, no entanto, depende de seqüenciamento e análise filogenética desses pares de vírus.

Nos casos das amostras EMP-2 e 5 e INTA 4, os vírus foram isolados do sangue de bezerros saudáveis de rebanhos com problemas reprodutivos, durante triagem para detectar animais PI. Como então explicar a presença de pares de vírus $\mathrm{cp} / \mathrm{ncp}$ antigenicamente semelhantes (ou idênticos) no sangue desses animais, se amostras cp/ncp têm a sua gênese determinada e restringem-se basicamente à casos de DM? Pelo menos duas hipóteses são possíveis: 1) os vírus cp foram gerados em outros animais do rebanho (possivelmente animais PI que geraram o cp a partir do ncp) e, eventualmente, transmitidos aos animais testados; 2) os animais testados eram PI, e a mutação do ncp originando o cp ocorreu pouco tempo antes da coleta das amostras. Nesses casos, esses animais ainda não teriam desenvolvido a DM e poderiam permanecer sem manifestar a enfermidade por algum tempo, apesar da presença de vírus dos dois biotipos. Nesse sentido, tem sido demonstrado experimentalmente que o desencadeamento da DM a partir da inoculação de animais PI com vírus cp pode levar várias semanas, até meses, período no qual os vírus cp e ncp replicam no animal (BAKER, 1995; BOLIN, 1995; FRAY et al., 1998). Em infecções naturais, a geração do vírus cp em um animal PI poderia ser seguida da replicação dos dois vírus durante algum tempo antes do desenvolvimento da enfermidade. A comprovação de que esses vírus ncp e cp, isolados de animais clinicamente saudáveis, constituem-se em verdadeiros pares poderá abrir uma nova perspectiva no estudo da gênese dos vírus cp e na patogenia das enfermidades associadas ao BVDV. O isolamento de vírus cp e ncp, antigenicamente relacionados, de fetos bovinos, a exemplo do que ocorreu com a amostra 34P, tem sido esporadicamente relatado. Nesses casos, os vírus cp, provavelmente, originamse do ncp, a exemplo do que ocorre nos casos de DM, constituindo o que tem sido denominado de "doença das mucosas fetal" (DUBOVI, E., informação pessoal).

Após a obtenção das populações puras de vírus cp e ncp, as amostras originais e os clones foram analisados individualmente quanto aos padrões de expressão das proteínas NS23 e NS3. O fenótipo cp do BVDV tem sido associado à habilidade do vírus expressar um polipeptídeo de massa aproximada de $80 \mathrm{kDa}$, denominada de NS3, embora o papel dessa proteína na produção de citopatologia permaneça incerto (DONIS, 1995). A análise da expressão desses polipeptídeos em células infectadas com as amostras de campo revelou invariavelmente duas bandas de reatividade, correspondentes aos polipeptídeos NS23 e NS3 da cepa Singer (figura 1A; 2B; 2C e 2D, linha 3). Os clones ncp apresentaram apenas uma banda de reatividade, com migração correspondente à NS23 da cepa Singer (figura 1A; $1 \mathrm{~B}$; $1 \mathrm{C}$ e $1 \mathrm{D}$, linha 5 e figura $2 \mathrm{~A}$, linhas 3 a 9). Uma variação significativa na migração da NS23 foi observada na amostra EMP-2 ncp em comparação com os outros vírus (figura 2A, linha 3 ). Por outro lado, os vírus cp apresentaram uma banda de reatividade adicional, correspondente à NS3 da cepa Singer (figura 1A; 1B; $1 \mathrm{C}$ e 1D, linha 4 ; figura 2B, linhas 3 a 9). Alguns vírus cp apresentaram alterações de migração da NS23 (figura 1A, linha 4; figura 2B, linha 3). Os clones cp da amostra 34P, por exemplo, apresentaram a banda correspondente à NS23 com uma migração maior (massa menor) do que as 
Tabela 2- Reatividade de um painel de anticorpos monoclonais com os clones de vírus citopáticos e não-citopáticos obtidos de amostras citopáticas do $\mathrm{BVDV}^{\mathrm{a}}$.

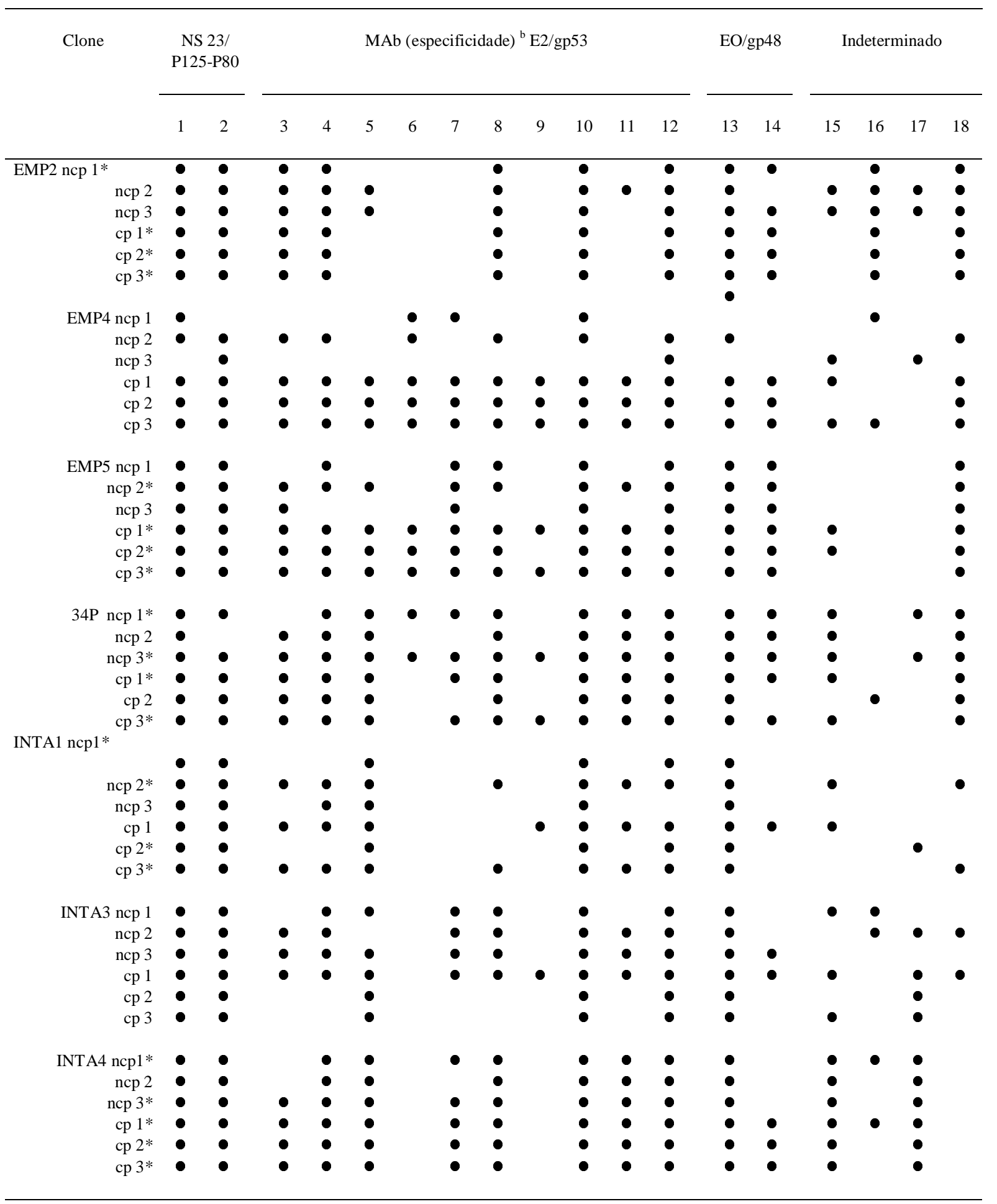

a - A reatividade dos MAbs foi testada por imunofluorescência indireta (IFI), utilizando cada MAb como anticorpo primário. Os pontos indicam reação positiva -

b Anticorpos monoclonais produzidos contra a cepa Singer (CORAPI et al., 1990). 1 - 20.10.6; 2 - 15.14.5; 3 - 12G4; 4 - 19F7; 5 - 20g7; 6 - 6d11; 7 - 10f9; 8 - 18d4; 9 - 4d1; 10 - 7.1.8; 11 - 26c6; 12 - 19F9; 13 - 15C5; 14 - 3D8; 15 - 3C6; 16 - 6C5; 17 - 32B3; 18 - 2D5.

Ciência Rural, v. 30, n. 1, 2000. 
demais (figura 1A, linha 4; figura 2B, linha 5). Os clones cp da amostra EMP-2, além da NS3 com migração normal, apresentaram duas bandas de reatividade próximas, mas não idênticas, à migração esperada para a NS23 (figura 2B, linha 3). Essas alterações de massa molecular da NS23 podem indicar a presença de deleções, rearranjamentos e/ou inserções no genoma desses vírus (MEYERS $\boldsymbol{e t}$ al., 1992; DONIS, 1995)

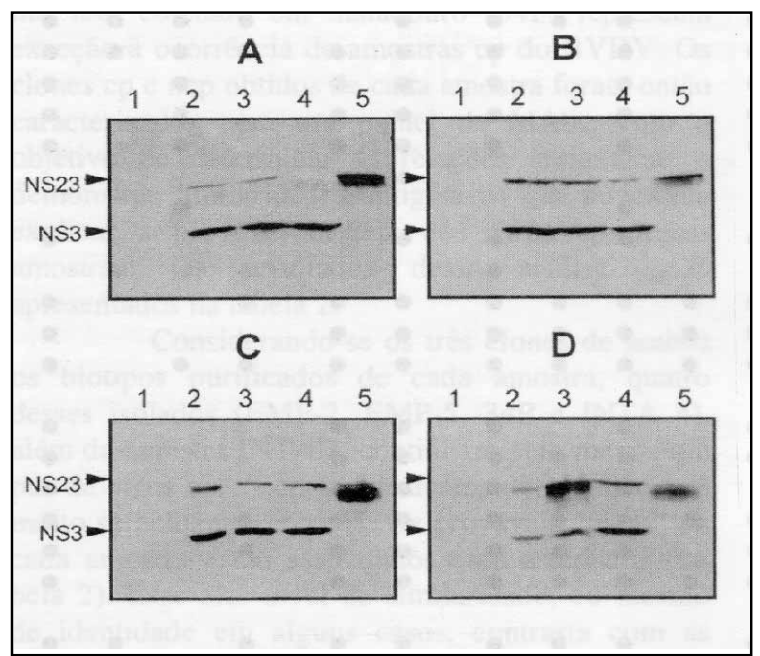

Figura 1 - Western immunoblot de lisados de células infectadas com as amostras de campo e com os clones cp e ncp obtidos a partir dessas amostras. A. Amostra 34P; B. INTA 3; C. EMP-5; D.EMP-4. Linha 1. Lisado de células MDBK não infectadas; linhas 2 a 5: lisados de células MDBK infectadas com a cepa Singer (2), isolado de campo (3), clone cp (4) e clone ncp (5), respectivamente. As pontas de seta indicam as posições das proteínas não-estruturais NS23 e NS3 da cepa Singer.

Os resultados desse estudo confirmam achados anteriores de que amostras citopáticas do BVDV contém uma mistura de vírus cp e ncp antigenicamente muito semelhantes . Adicionalmente, o presente estudo demonstrou que amostras citopáticas contendo os dois biotipos não se restringe a casos de DM e podem ocasionalmente ocorrer em outras condições associadas à infecção pelo BVDV. Dentre as amostras analisadas, duas apresentam alterações significativas na migração da NS23, podendo indicar a presença de inserções, deleções ou duplicações no genoma que levem à expressão da NS3. O mecanismo de geração da NS3 nessas amostras constitui-se em objeto para futuras investigações, que poderão trazer contribuições significativas para um melhor conhecimento da biologia e patogenia das infecções pelo BVDV.

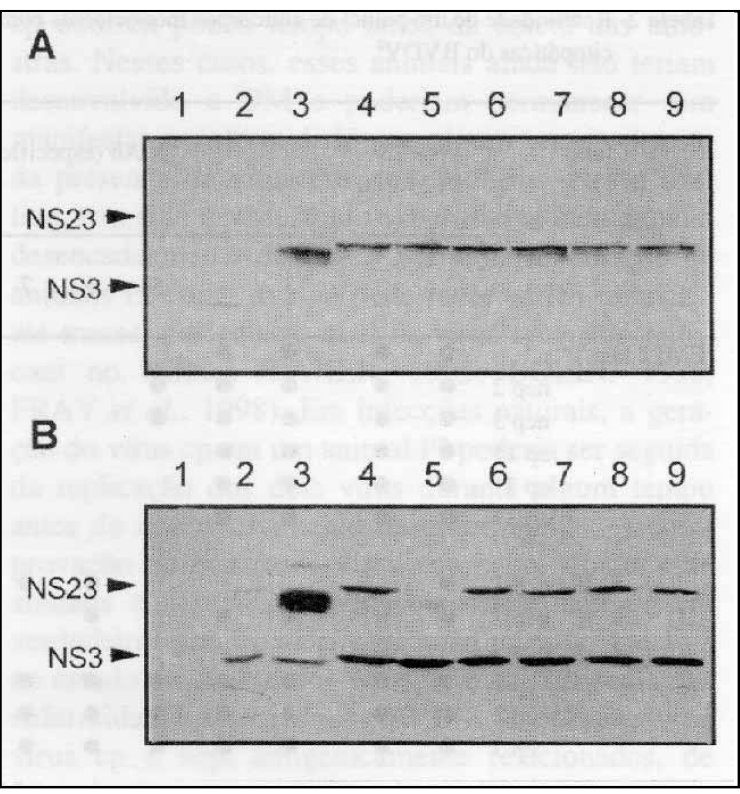

Figura 2 - Western immunoblot de lisados de células infectadas com os clones não-citopáticos e citopáticos obtidos a partir das amostras. A. Clones não-citopáticos. Linhas: 1. Células MDBK não-infectadas; 2. Células MDBK infectadas com a cepa Singer. 3 EMP-2.2ncp 4. EMP4.6ncp; 5. EMP-5.3ncp; 6. INTA-1.3ncp; 7. INTA 3.1ncp; 8. INTA 4.3ncp. 9; 34P-2ncp. B. Clones citopáticos. Linha 1. Células MDBK não-infectadas; 2. Células MDBK infectadas com a cepa Singer. 3. EMP$2.3 \mathrm{cp} ;$ 4. INTA $3.4 \mathrm{cp} ; 5$. 34P-4cp; 6. INTA $4.3 \mathrm{cp} ; 7$. EMP-4.2cp; 8. EMP-5.3cp; 9.INTA $1.3 \mathrm{cp}$. As pontas de seta indicam as posições das proteínas nãoestruturais NS23 e NS3 da cepa Singer.

\section{INFORMAÇÃO PESSOAL}

Dr. Edward Dubovi. Veterinary Diagnostic Laboratory, Cornell University, Ithaca, NY.

\section{REFERÊNCIAS BIBLIOGRÁFICAS}

BAKER, J.C. The clinical manifestations of bovine viral diarrhea infection. Vet Clin North America, v. 11, p. 425-446, 1995.

BOLIN, S.R. The pathogenesis of mucosal disease. Vet Clin North America, v. 11, p. 489-500, 1995.

BOLIN, S.R., RIDPATH, J.F. Prevalence of bovine viral diarrhea genotypes and antibodies against those viral genotypes in fetal bovine serum. J Vet Diagn Invest, v. 10, p. 135-139, 1998.

BOTTON, S.A, GIL, L.H.V.G., SILVA, A.M. et al., Caracterização preliminar de amostras do vírus da diarréia viral bovina (BVDV) isoladas no Brasil. Pesq Vet Bras, v. 18, p. 84-92, 1998.

BROWNLIE, J. The pathogenesis of bovine viral diarrhea vírus infections. Vet Sci Tech, OIE, v. 9, p. 43-59, 1990. 
COLlETT, M.S., MOENNIG. V., HORZINEK, M. Recent advances in pestivirus research. J Gen Virol, v. 70, p. 253$266,1989$.

CORAPI, W.V., DONIS, R., DUBOVI, E.J. Monoclonal antibody analyses of cytopathic and noncytopathic viruses from fatal bovine viral diarrhea virus infection. J Virol, v. 62, p. 28232827, 1988.

CORAPI, W.V., DONIS, R.O., DUBOVI, E.J. Characterization of a panel of monoclonal antibodies and their use in the study of the antigenic diversity of bovine viral diarrhea virus. Am J Vet Res, v. 51, p. 1388-1394, 1990

DONIS, R. Molecular biology of bovine viral diarrhea virus and its interactions with the host. Vet Clin North America, v. 11, p. 393-421, 1995 .

DUBOVI, E.J. Bovine viral diarrhea virus. In: SIMPÓSIO INTERNACIONAL SOBRE HERPESVÍRUS BOVINO E DIARRÉIA VIRAL BOVINA. Santa Maria, RS. Anais... Santa Maria: Universidade Federal de Santa Maria, 1998, p. 119.

FRANCKI, R., FAUQUET, C., KNUDSON, D. et al. Classification and nomenclature of viruses. Wien-New York: SpringerVerlag. Fifth Report on the International Committee on Taxonomy of Víruses (ICTV), v. 2, Arch Virol[Suppl.2]. Vienna, N. York: Springer-Verlag, 1991.

FRAY, M.D., CLARKE, M.C., THOMAS, L.H. et al. Prolonged viral shedding and viraemia of cytopathogenic bovine virus diarrhea virus in experimental late-onset mucosal disease. Vet Rec, 143:608-611, 1998

GILLESPIE, J.H., MADIN, S.H., DARBY, N.B. Cellular resistance in tissue culture induced by noncytopathogenic strains to a cytopathogenic strain of virus diarrhea virus of cattle. Proc
Soc Exp Biol Med, v. 110, p. 248-250, 1962.

HORZINEK, M.C. Non-arthropod-borne togaviruses. New York: Academic, 1981. 200 p.

HOWARD, C.J., CLARKE, M.C., BROWNLIE, J. Comparisons by neutralisation assay of pairs of non-cytopathogenic and cytopathogenic strains of bovine virus diarrhoea virus isolated from cases of mucosal disease. Vet Microbiol, v. 13, p. 361$369,1987$.

LAEMNLI, V.K. Cleavage of structural proteins during the assembly of the head of bacteriophage T4. Nature, v. 227, p. 680-685, 1970.

McCLURKIN, A.W., BOLIN, S.R., CORIA, M.F. Isolation of cytopathic and noncytopathic bovine viral diarrhea vírus from the spleen of cattle acutely and chronically affected with bovine viral diarrhea. J Am Vet Med Assoc, v. 186, p. 568-575, 1985 .

MENDEZ, E., RUGGLI, N., COLLETT, M. et al. Infectious bovine viral diarrhea virus (strain NADL) RNA from stable cDNA clones: a cellular insert determines NS3 production and viral cytopathogenicity. J Virol, v. 72, p. 4737-4745, 1998.

MEYERS, G., TAUTZ, N., STARK, R. Rearrangement of viral sequences in cytopathogenic pestivíruses. Virology, v. 191, p. 368-376, 1992.

RIDPATH, J.F., LEWIS, T.L., BOLIN, S.R. et al. Antigenic and genomic comparison between non-cytopathic and cytopathic bovine viral diarrhea viruses isolated from cattle that had spontaneous mucosal disease. J Gen Virol, v. 72, p. 725-729, 1991.

Ciência Rural, v. 30, n. 1, 2000. 